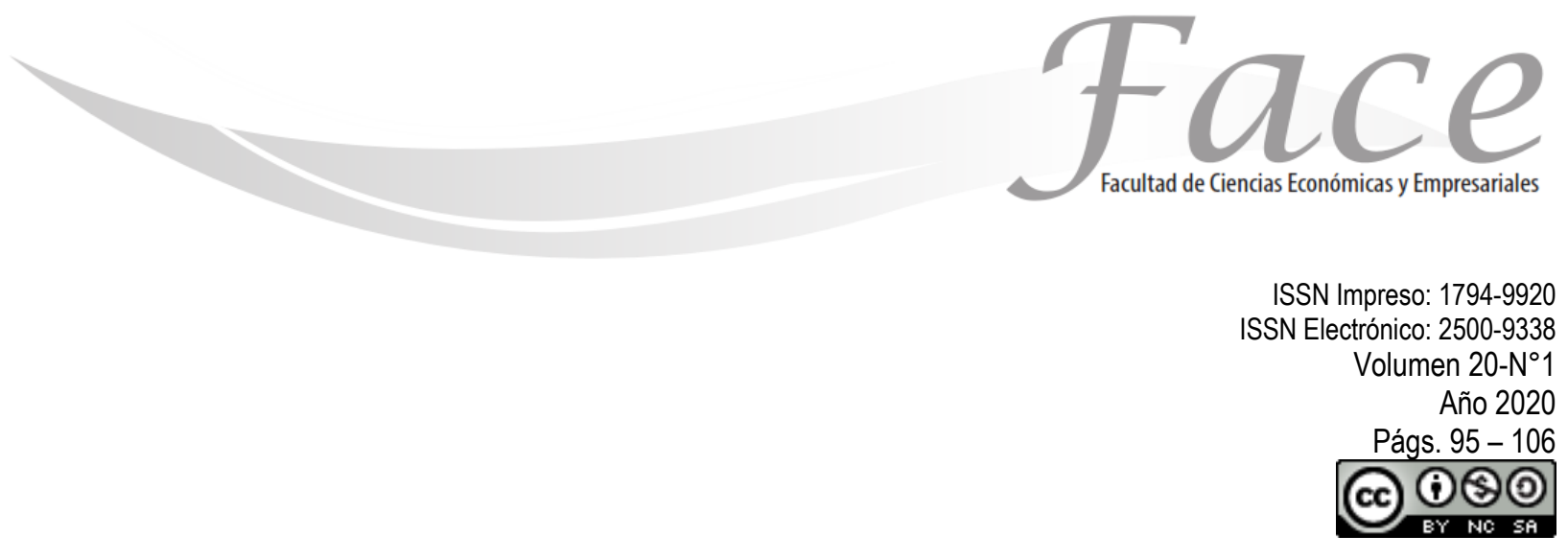

\title{
MOVILIDAD E INCREMENTO EN CASOS CONFIRMADOS DE COVID-19: RELACIÓN ECONOMÉTRICA EN LAS ENTIDADES FEDERATIVAS DE MÉXICO
}

\author{
Zeus Salvador Hernández Veleros * \\ ENLACE ORCID: https://orcid.org/0000-0002-3162-9122 \\ Daniel Velázquez Orihuela ** \\ ENLACE ORCID: https://orcid.org/0000-0002-0953-9905
}

Fecha de Recepción: Septiembre 27 de 2020

Fecha de Aprobación: Diciembre 3 de 2020

\section{Resumen:}

El objetivo de este documento es cuantificar la relación entre movilidad y contagios de COVID-19 para las entidades de México, para lo cual consideramos el cambio en el número de contagiados entre el 12 de abril y el 12 de mayo en las entidades mexicanas en función del número inicial de confirmados y el índice de movilidad ponderado por el parque vehicular estatal. La relevancia de esto radica en el fin de la Jornada de Sana Distancia con fecha 30 de mayo, ello puede traer consecuencias en el aumento en el número de contagios y, por ende, en el número de defunciones. En segundo lugar, proponemos cómo financiar un período de aislamiento severo y un aumento en el gasto público una vez que concluya tal medida, gracias a un crédito a cubrir posteriormente con recursos de los remanentes de Banco de México.

Palabras clave: Movilidad, COVID-19, Relación econométrica, Jornada Sana Distancia, Remanentes de Banxico

\footnotetext{
* Doctor en Economía. Profesor-investigador del Instituto de Ciencias Económico Administrativas. Universidad Autónoma del Estado de Hidalgo, México. Contacto: zeus_hernandez@uaeh.edu.mx

* * * Doctor en Economía Profesor-investigador del Instituto de Ciencias Económico Administrativas. Universidad Autónoma del Estado de Hidalgo, México. Contacto: daniel velazquez7607@uaeh.edu.mx

${ }^{* * * *}$ Maestro en educación adscrito a la Coordinación Académica Región Altiplano. Universidad Autónoma de San Luis Potosí. México. Correo electrónico: marcos.martinez@uasIp.mx
} 


\title{
MOBILITY AND INCREASE IN CONFIRMED CASES OF COVID-19: ECONOMETRIC RELATIONSHIP IN THE STATES \\ OF MEXICO
}

\begin{abstract}
:
Our aim of this document is to quantify the relationship between mobility and COVID-19 infections for Mexican states, we consider the change in the number of infected between April 12 and May 12 in Mexican states based on of the initial number of confirmed and the mobility index weighted by the number of cars in each state. The relevance of this study is related to the end of the Jornada de Sana Distancia on May 30, which may have consequences in the increase in the number of infections and, therefore, in the number of deaths. Second, we propose how to finance a period of severe isolation and an increase in public spending once this measure is concluded, thanks to a loan to be covered later with resources from Banco de México's remnants.
\end{abstract}

Keywords: Mobilitity, COVID-19, Econometric relationship, Jornada Sana Distancia, Remanentes de Banxico

\section{MOBILIDADE E AUMENTO NOS CASOS CONFIRMADOS DA COVID-19: RELAÇÃO ECONOMÉTRICA NOS ESTADOS DO MÉXICO}

\begin{abstract}
Resumo:
Nosso objetivo com este documento é quantificar a relação entre mobilidade e infecções por COVID-19 para estados mexicanos, consideramos a mudança no número de infectados entre 12 de abril e 12 de maio nos estados mexicanos com base no número inicial de confirmados e na mobilidade. índice ponderado pela quantidade de carros em cada estado. A relevância deste estudo está relacionada ao fim da Jornada de Sana Distancia em 30 de maio, o que pode ter consequências no aumento do número de infeç̧ões e, portanto, do número de óbitos. Em segundo lugar, propomos como financiar um período de severo isolamento e um aumento dos gastos públicos uma vez concluída esta medida, graças a um empréstimo a ser coberto posteriormente com recursos do remanescente do Banco de México.
\end{abstract}

Palavras-chave: Mobilidade, COVID-19, Relação econométrica, Jornada Sana Distancia, Remanentes de Banxico 


\section{INTRODUCCIÓN:}

Para entender la relación entre movilidad y contagios de COVID-19 en las entidades de México planteamos un modelo econométrico que relaciona tales elementos ponderados por el número de vehículos. El cual muestra que es necesario el aislamiento para poder vencer los contagios, ya que la movilidad es un determinante de la velocidad de la epidemia, al ser las personas quienes contagian a otras, las cuestiones de transporte en vehículos o peatonal conforman el canal de conducción, ya que no es posible mantener la sana distancia en el transporte público y las relaciones persona a persona son impulsores de tal fuerza de contagio.

Los estudios más recientes indican que tal vez el virus SARS-CoV-2, que provoca la enfermedad COVID-19, inició en noviembre de 2019, con reportes del gobierno de China en diciembre de 2019. El primer caso de esta enfermedad en México se registró oficialmente el 27 de febrero y al 30 de septiembre de 2020 , casi seis meses después, se reportaron 64,158 muertes acumuladas.

Este documento está conformado por un apartado en el cual establecemos algunos de los estudios realizados en torno a la relación entre movilidad y contagios en espacios geográficos específicos de Italia y China. Posteriormente, en un apartado metodológico, presentamos cuestiones metodológicas referentes a la movilidad en México y en sus entidades, a los contagios del COVID-19, a las muertes y a las políticas públicas implementadas en México. A continuación, en el apartado de resultados, estimamos la relación entre movilidad, confirmados con covid-19 y cambio en el número de casos confirmados por entidad; asimismo, presentamos una propuesta sobre cómo financiar una política de confinamiento y un posterior apoyo a la demanda agregada. Para finalizar presentamos algunas conclusiones.

\section{MARCO TEÓRICO:}

En un tema que a lo sumo tiene nueve meses, es difícil encontrar en revistas internacionales documentos que reflejen un análisis profundo y extenso del tema en cuestión, en este caso sobre la relación entre movilidad y contagios de SARS-CoV-2 en espacios geográficos específicos. Uno de ellos es el desarrollado por Carteni et al. (2020) referente a cómo la movilidad de los habitantes de Italia influyó en la velocidad de la pandemia COVID-19 en diversas regiones y provincias de esa nación, para ello plantearon un modelo econométrico de los casos diarios confirmados de esta enfermedad entre el 21 de febrero y el 5 de mayo, contra variables cómo los hábitos de movilidad detectados por el Ministerio de Transporte en el periodo de enero a mayo de 2020, la densidad de la población en las capitales de sus provincias relativa al año 2019, la contaminación por partículas medida en 2019 por la Agencia Italiana Regional para la Protección Ambiental para el número de días en que se superó el límite diario, (ponderada por la población), la temperatura diaria medida de enero a mayo de 2020 (promedio de la temperatura observada $\mathrm{x}$ días antes del i-ésimo día con referencia a la t-ésima región), el número de pruebas por día realizadas a la población en cada región, el tiempo de viaje promedio ponderado de la región i-ésima hasta el clúster inicial de COVID19 en Codongo en el día i-ésimo, y las tasas de movilidad estimadas por Isfort (número promedio de personas de 14 a 80 años de edad que han realizado por lo menos un viaje $x$ días antes del i-ésimo día con respecto a la región.t-ésima), así como una constante. En sus hallazgos Carteni et al. (2020) encuentran que la densidad poblacional, la contaminación y el número de pruebas por día tienen una relación directa con la infección; en tanto que, la temperatura tiene una relacion inversa con la velocidad del virus; así como que las regiones más cercanas al punto de inicio del contagio tienen un riesgo más alto. 
Xie et al. (2020) emplearon un análisis exploratorio espacial y un método geodetector para estudiar las características de diferenciación espacial y temporal, así como los factores de influencia de la velocidad epidémica del COVID-19 en China, ello a partir de los casos confirmados, la temperatura promedio, y datos socioeconómicos. Cabe aclarar que el uso del número de casos acumulados de COVID-19 es una medida sesgada de la tasa de velocidad epidémica, debido a las diferencias en los tamaños poblacionales, por lo cual ese número fue dividido por el número de días empleado para calcular la tasa mencionada.

Estos autores utilizaron el método exploratorio de análisis de datos espaciales para verificar si el valor observado de una unidad tiene correlación espacial con los valores observados de sus unidades vecinas. El índice I de Moran global se utiliza para medir la correlación espacial global, mientras que el índice I de Moran local en LISA (indicadores locales de asociación espacial) fue usado para medir la correlación espacial local. Dado que la diferenciación espacial es una característica básica de los fenómenos geográficos, el método de Geodetector puede medir el grado de heterogeneidad estratificada espacial y probar su significancia, a través de que la varianza al interior de los estratos sea menor que la varianza entre estratos. El método de Geodetector consta de cuatro módulos: detección de factores, detección de interacciones, detección de riesgos y detección ecológica.

El tercer aspecto desarrollado en el artículo comentado fue referente a los factores que influyen en la velocidad de la epidemia, para ello consideraron que la gente ha sido el transportista, la red de transporte ha sido el canal, las conexiones sociales y económicas han sido la fuerza impulsora interna en el proceso de propagación de la epidemia. Por lo tanto, seleccionaron los indicadores que reflejan la distribución de la población, el flujo de población de Wuhan, la accesibilidad del tráfico, la intensidad de la conexión económica, la temperatura promedio y las condiciones de las instalaciones médicas como factores de detección, y la tasa de expansión epidémica como factor de detección para evaluar el mecanismo de formación para el patrón espacial de la epidemia de COVID-19.

Las principales conclusiones de Xie et al. (2020) son: (1) la epidemia se propagó rápidamente del 24 de enero al 20 de febrero de 2020, y la distribución de las áreas epidémicas tendió a ser estable en el tiempo. La tasa de propagación de la epidemia en la provincia de Hubei, en sus alrededores y en algunas ciudades económicamente desarrolladas fue mayor, mientras que en la parte occidental de China y en áreas remotas del centro y este de China fue menor. (2) Las correlaciones espacial global y local de las características de la distribución epidémica presentan una correlación positiva; esto es, las características de correlación espacial global experimentaron un proceso de cambio de aglomeración a descentralización. Las características de correlación espacial local se componían principalmente de los tipos de agrupamiento "alto-alto" y "bajo-bajo", y la situación del trazado contiguo fue muy significativo. (3) La afluencia de población de Wuhan y la fuerza de conexión de la economía fueron los principales factores que afectaron la propagación de la epidemia, junto con la distribución de la población, la accesibilidad al transporte, la temperatura promedio y las instalaciones médicas, que afectaron la propagación de la epidemia en diversos grados.

(4) Los factores de detección interactuaron principalmente a través de la mejora mutua y la mejora no lineal, y su influencia sobre la tasa de propagación de la epidemia excedió la de los factores individuales. Además, cada factor de detección tiene un rango de intervalo que favorece la propagación de la epidemia.

\section{METODOLOGÍA:}

\section{Movilidad en México y en sus entidades}

Los contagiados con el virus SARS-CoV-2 pueden transmitir el mismo a otras personas, de ahí las políticas de aislamiento y de reducción de la movilidad, pero ello implica no sólo cuestiones de salud pública (contagiados, muertes) también económicas por sus efectos, pero las cuestiones económicas también repercuten en la movilidad y en la salud pública.

Apple Maps y Google Community Mobility Reports registran e informan la movilidad grupal de sus usuarios por países y subregiones, el primero lo divide en función del medio utilizado: conducción de un vehículo particular (driving), caminata (walking) y 
transporte público (transit). Por su parte, Google presenta datos al considerar el lugar de destino del movimiento: venta al por menor y recreación, comestibles y farmacias, parques, estaciones de tránsito de transporte público, lugares de trabajo y residencial. En el caso de México está información es heterogénea por entidades federativas.

De acuerdo con los datos de conducción (driving) de coches particulares Apple considera un índice de movilidad que tiene un valor de 100 el día 13 de enero del 2020, para México tal índice registró el máximo de movilidad el 14 de febrero (152.62), día del amor y la amistad; el mínimo ocurrió dos meses después, el 12 de abril (32.18), ya en medio de las medidas para disminuir los contagios; no obstante, desde este mínimo se observa una tendencia hacia el alza hasta el último dato disponible del nueve de abril (63.08), el aumento es de 30.9 , con obvias implicaciones en los contagios (Figura 1).

Otro aspecto a destacar es que existe una estacionalidad semanal: los días sábado y domingo la movilidad es mayor. Asimismo, la caída en la movilidad se presenta desde el día 16 de marzo (80.27), cuando fue registrado el índice más bajo hasta esa fecha, antes del inicio de las políticas públicas del gobierno federal (Figura 1).

Figura 1. Movilidad de la conducción en México, índice (100 = 13 de enero de 2020), 13/01 a 09/05

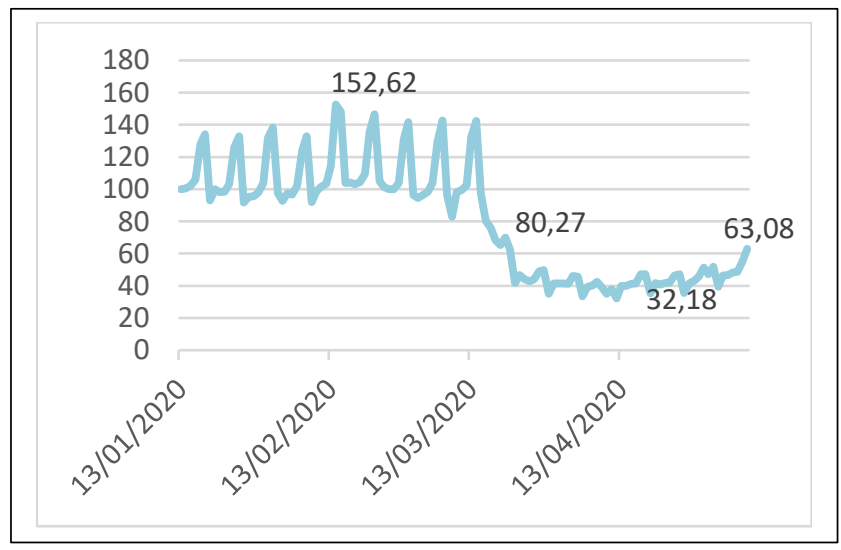

Fuente: elaboración propia a partir de Google Maps.

La política pública para disminuir la movilidad "Quédate en casa" inició el 23 de marzo. La "Jornada Nacional de Sana Distancia" fue anunciada el 14 de marzo para entrar en vigor el 23 de tal mes, pero ante el hecho de que gran parte de la sociedad ya había iniciado un auto confinamiento, sin mediar las indicaciones de la autoridad, el gobierno federal anunció la aplicación inmediata de tales medidas el jueves 19 (el índice tal día fue 65.28); de igual manera, fue anunciado el 16 de abril que esta jornada concluirá el 30 de mayo.

El último pico más alto se presentó el fin de semana largo que inició el sábado 14 de marzo; 13 entidades federativas presentaron el máximo índice de movilidad del año en esa fecha. El 12 de abril no sólo el país registró el mínimo índice de movilidad en todo el periodo de cobertura de los datos (32.18), 18 entidades también presentaron sus respectivos mínimos, pero desde ese momento hasta el nueve de mayo han presentado aumentos las 32 subdivisiones territoriales, con aumentos en el valor del índice tan altos como los de Coahuila (85.96), Tamaulipas (67.15), Sinaloa (63), Sonora (59) y Colima (56) (Figura 2).

Figura 2. Cambio en la movilidad por entidad federativa del 12 de abril al 9 de mayo (cambio en el índice)

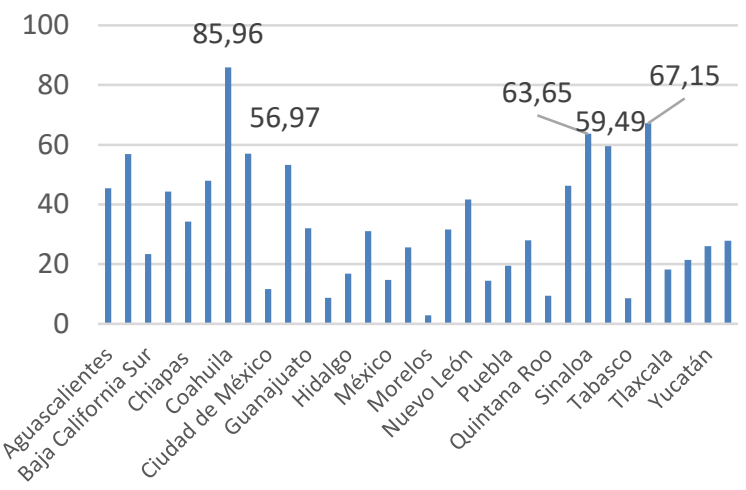

Fuente: elaboración propia a partir de Google Maps.

De esta manera, si consideramos el registro que indica propiamente una dinámica de descenso notoria, que es del día 16 de marzo, 80.27, hasta el mínimo de la movilidad, 12 de abril, 32.18, tenemos 28 días de tendencia descendente; si comparamos aquel dato mínimo con el último dato disponible del día 9 de mayo, 63.08 , encontramos una tendencia ascendente.

Uno de los argumentos de porque las personas no se quedan en casa es que viven al día, tal vez eso no sea tan correcto para los usuarios de un equipo de Apple. Si consideramos los datos de Google Community Mobility Reports de los seis índices por destino en un 
único índice (14 de febrero es la base igual a 1), como un promedio simple, encontramos que desde el $15 \mathrm{de}$ marzo el índice inició un descenso, el cual llegó a un mínimo el 10 de abril (0.53), pero desde esa fecha ha ascendido, con dos registros máximos: uno el 30 de abril (0.66) y otro el 9 de mayo (0.66), último dato disponible (Figura 3).

Figura 3. Movilidad total a seis destinos en México,

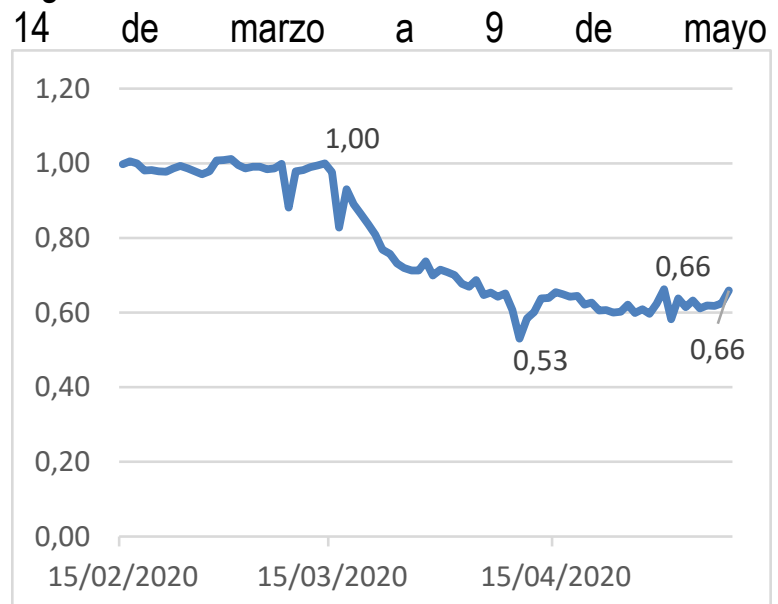

Fuente: elaboración propia a partir de Google Community Mobility Reports.

Por lugar de destino al cual se dirigen las personas, si definimos un índice con valor a 1 para el registro del 14 marzo encontramos para el 9 de mayo que el mayor descenso (0.57) se registra en el destino "Ventas al por menor y recreación", con un valor de índice de 0.43 ; el segundo descenso más grande (0.55) se registra en los destinos "Parques", el índice fue 0.45; la tercer caída más grande $(0.53)$ es hacia "Estaciones de tránsito", el índice es 0.47 ; en cuarto lugar está el descenso (0.30) que se presentó en el destino "Comestibles y farmacias", (índice 0.70); por último, "Lugares de trabajo", es el destino con menor reducción $(0.25)$, pues su índice es 0.75 . El destino residencia presentó un aumento es este periodo: de 1.00 a 1.18 (Figura 4).

El día 10 de abril se registró el mínimo índice en tres destinos: "Ventas al por menor y recreación" (0.28), "Estaciones de tránsito" (0.27) y "Lugares de trabajo" (0.27), hasta el 9 de mayo la tendencia ha sido al alza; los aumentos respectivos son: $0.15,0.15$ y 0.43 . Los otros dos destinos: "Comestibles y farmacias" y "Parques", tuvieron su mínimo valor el 26 de abril: 0.63 y 0.38 , respectivamente, para el 9 de mayo los índices respectivos aumentaron en 0.13 y 0.08 , para llegar a 0.76 en "Comestibles y farmacias" y 0.46 en el destino "Parques". "Residencial observó su máximo el 10 de abril (1.31) y el 9 de mayo había descendido en 0.13 , a un índice de 1.18(Figura 4).

Figura 4. Movilidad a seis destinos en México, índices al 14 de marzo iguales a 1, 14 de marzo a 9 de mayo

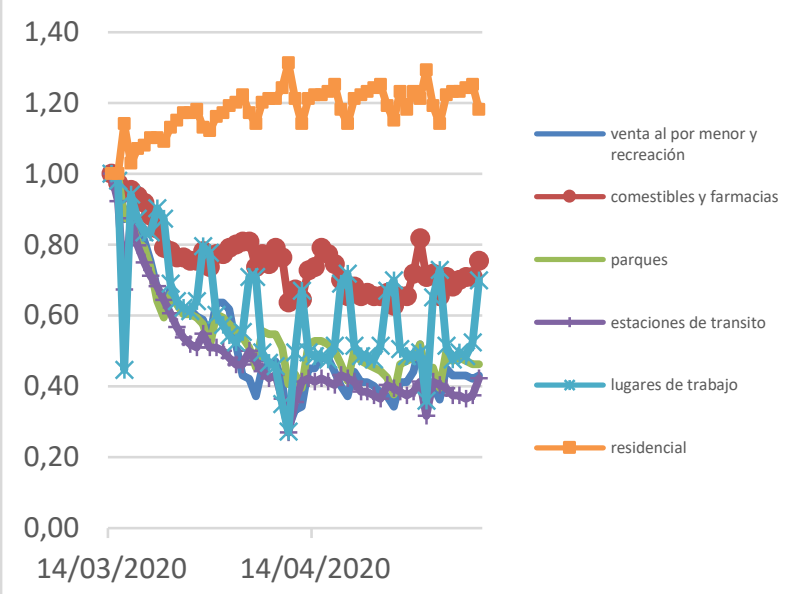

Fuente: elaboración propia a partir de Google Community Mobility Reports.

Como hemos indicado antes, para los datos de Apple existe un patrón distinto por tipo de día de la semana: laborales y no laborales, lo mismo ocurre con los datos de Google por destino. Entre el primer periodo de días laborales (17 a 20 de marzo) y el último considerado (4 a 8 de mayo) se observa un descenso en el promedio de cinco destinos, excepto en el "Residencial" (Figura 5); lo mismo ocurre entre el primer periodo de días no laborales (14 a 16 de marzo) y el último período de días no laborales (2 a 3 de mayo); igual quien muestra una dinámica distinta es "Residencial" (Figura 6).

Figura 5. Movilidad a seis destinos en México en días laborales, 17-20 de marzo a 4-8 de mayo

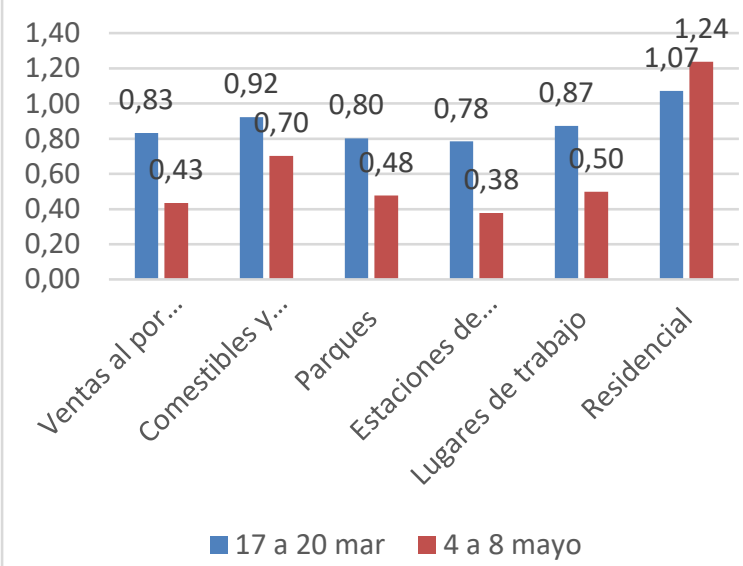

Fuente: elaboración propia a partir de Google Community Mobility Reports. 
Figura 6. Movilidad a seis destinos en México en días no laborales, 14-16 de marzo a 2-3 de mayo

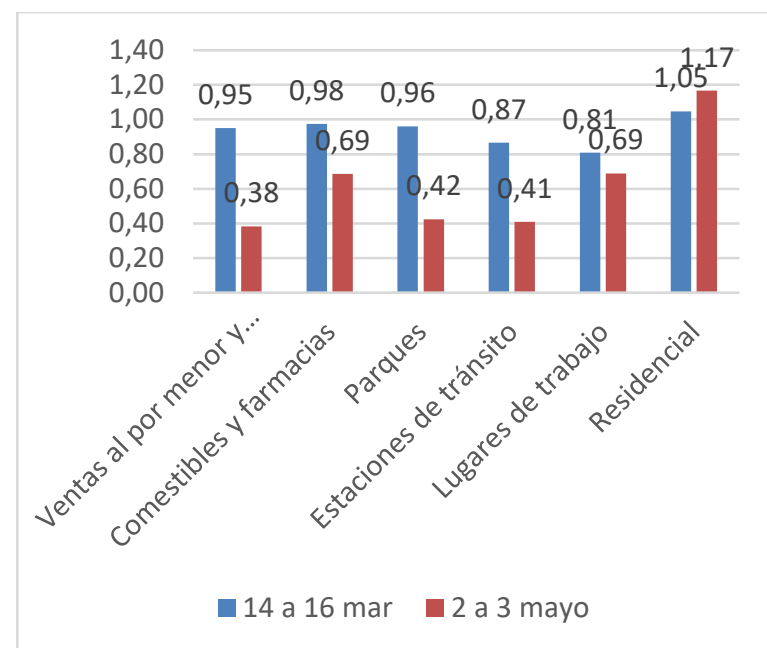

Fuente: elaboración propia a partir de Google Community Mobility Reports.

Si consideramos los desplazamientos al lugar de trabajo encontramos una estacionalidad semanal inesperada: los días domingos y sábados encontramos la máxima movilidad; por ejemplo, domingos 12 de abril (69), 19 de abril (74), 26 de abril (72), 3 de mayo (75), sábado 9 de mayo (72); por otra parte, los valores mínimos se registraron días como el lunes 16 de marzo (46), viernes 10 de abril (28) y vienes $1^{\circ}$ de mayo (37). Lo anterior se puede deber a que muchas personas regresan a sus lugares de trabajo los domingos, o bien tienen actividades laborales los días de fin semana (Figura 7).

Figura 7. Movilidad al destino trabajo en México, 14 de marzo a 9 de mayo

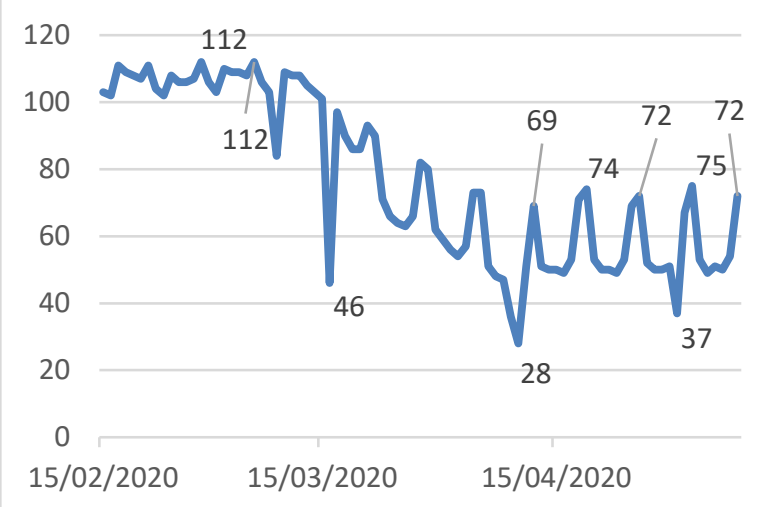

Fuente: elaboración propia a partir de Google Community Mobility Reports.

\section{Contagios, muertes por covid-19 y fin de la Jornada Nacional de Sana Distancia}

El 16 de abril se anunció por parte del Subsecretario Prevención y Promoción de Salud, Hugo López-Gatell, que la Jornada Nacional de Sana Distancia concluirá el 30 de mayo, para reanudar actividades el primero de junio; pero en 979 municipios, donde la incidencia es menor, la reclusión cesará el 17 de mayo, a lo anterior agregó que el pico máximo de expansión de la enfermedad se espera entre el 8 y el 10 de mayo y que es posible que el 25 de junio se declara el fin del primer ciclo, al agotarse el 95\% de los casos esperados. El 5 de mayo anunció el funcionario referido que el máximo de contagios se alcanzaría el 8 de mayo y no el 5 como había pronosticado antes.

Con respecto a las anteriores declaraciones, las cifras indican un aumento más grave en el número de confirmados que el estimado: el 8 de mayo la cifra de nuevos casos confirmados fue 1,982 casos, en los 12 días siguiente ha sido superada tal cifra en siete ocasiones; la cifra del 20 de mayo es la más alta: 2,713 personas (Figura 8). Si consideramos desde el primer día que se registraron más de 1,000 casos, lo cual ocurrió el 23 de abril, encontramos que al 20 de mayo el aumento en el número de personas confirmadas es de $160.11 \%$ (Figura 8).

Figura 8. Personas confirmadas con Covid-19 en México, 23 de abril a 20 de mayo.

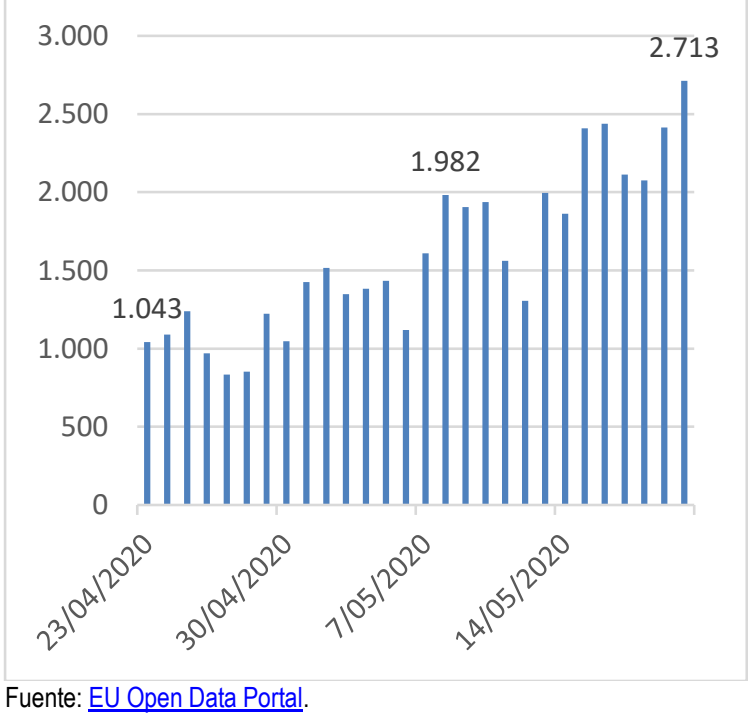


Con respecto a las defunciones tenemos que el 8 de mayo la cifra fue 252; en los 12 días siguientes a tal fecha se registraron cinco días con cifras de defunciones mayores a las del 8 de mayo. Así, el número de defunciones entre el 20 de mayo y el 23 de abril aumentó 195.57 \% (Figura 9).

Figura 9. Decesos por Covid-19, México, 23 de abril a 20 de mayo.

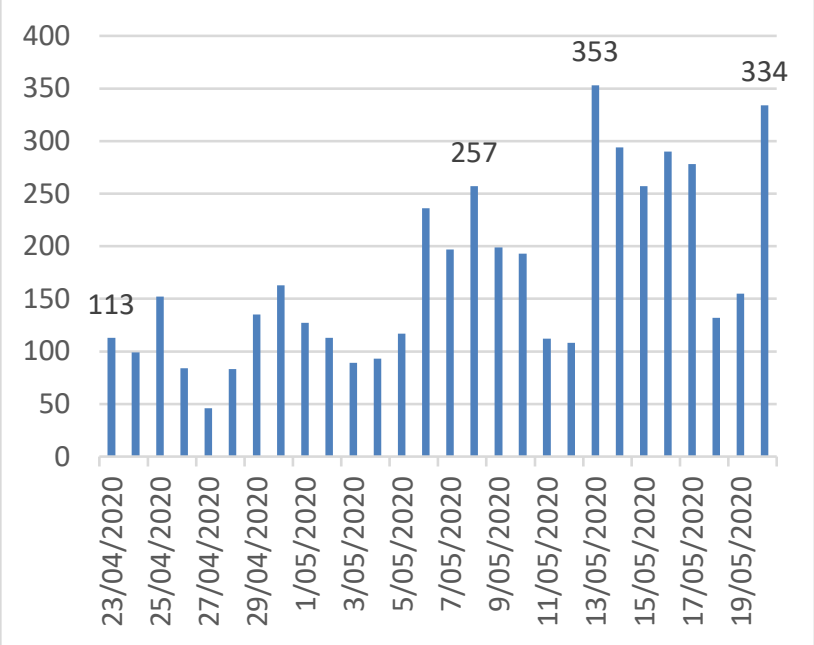

Fuente: EU Open Data Portal.

En conclusión, el número de confirmados y de muertes aumentó después del 8 de mayo, no descendió. La política de conclusión de la sana distancia está en entredicho por las mismas cifras oficiales; no es necesario recurrir a otras cifras con más muertes o con factores de expansión para encontrar contradicciones.

\section{RESULTADOS:}

Para determinar la relación entre movilidad, número inicial de confirmados y el cambio en el número de casos confirmados estimaremos una regresión para las entidades de México. Nuestros datos a considerar son: casos confirmados de Covid-19 el 12 de abril (Confirmados12abr), casos confirmados de Covid-19 el 12 de mayo (Confirmados12may), movilidad del 12 de abril según Apple Maps (Movilidadini12abril), número de vehículos en 2018 (Vehículos2018), movilidad 12 de abril ponderada por el número de vehículos (Moviponde12abril) (Tabla 1).

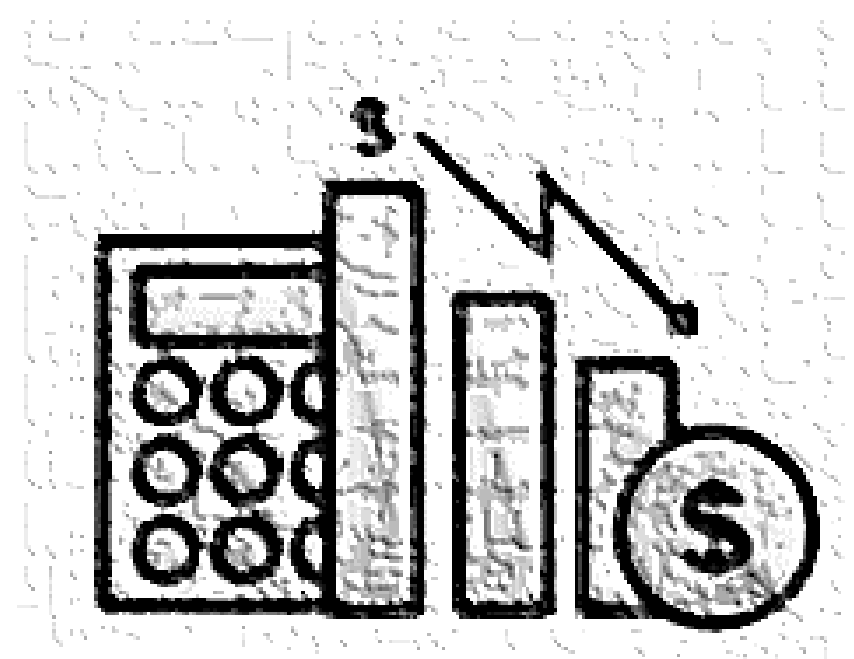


Tabla 1. Datos para regresión sobre la relación entre movilidad, confirmados con Covid-19 y cambio en el número de confirmados por entidad federativa Parte 1.

\begin{tabular}{|c|c|c|c|}
\hline Entidad & $\begin{array}{l}\text { Confirmados12 } \\
\text { abr }\end{array}$ & $\begin{array}{l}\text { Confirmados12 } \\
\text { may }\end{array}$ & Cambioconfirmados \\
\hline Aguascalientes & 55 & 382 & 327 \\
\hline Baja California & 335 & 2,576 & 2,241 \\
\hline Baja Cal. Sur & 120 & 401 & 281 \\
\hline Campeche & 19 & 211 & 192 \\
\hline Chiapas & 43 & 433 & 390 \\
\hline Chihuahua & 61 & 730 & 669 \\
\hline Coahuila & 157 & 584 & 427 \\
\hline Colima & 7 & 46 & 39 \\
\hline Cd. de México & 1,328 & 10,529 & 9,201 \\
\hline Durango & 16 & 123 & 107 \\
\hline Guanajuato & 82 & 544 & 462 \\
\hline Guerrero & 70 & 618 & 548 \\
\hline Hidalgo & 58 & 603 & 545 \\
\hline Jalisco & 156 & 670 & 514 \\
\hline México & 523 & 6,540 & 6,017 \\
\hline Michoacán & 61 & 610 & 549 \\
\hline Morelos & 44 & 874 & 830 \\
\hline Nayarit & 22 & 247 & 225 \\
\hline Nuevo León & 124 & 687 & 563 \\
\hline Oaxaca & 34 & 271 & 237 \\
\hline Puebla & 256 & 1,172 & 916 \\
\hline Querétaro & 61 & 273 & 212 \\
\hline Quintana Roo & 223 & 1,144 & 921 \\
\hline San Luis Potosí & 53 & 308 & 255 \\
\hline Sinaloa & 229 & 1,579 & 1,350 \\
\hline Sonora & 69 & 567 & 498 \\
\hline Tabasco & 153 & 1,876 & 1,723 \\
\hline Tamaulipas & 53 & 788 & 735 \\
\hline Tlaxcala & 40 & 398 & 358 \\
\hline Veracruz & 78 & 1,497 & 1,419 \\
\hline Yucatán & 107 & 883 & 776 \\
\hline Zacatecas & 15 & 160 & 145 \\
\hline Aguascalientes & 32.07 & 611,917 & 196,242 \\
\hline Baja California & 34.76 & $1,700,000$ & 590,920 \\
\hline Baja Cal. Sur & 21.83 & 510,615 & 111,467 \\
\hline Campeche & 40.67 & 325,505 & 132,383 \\
\hline Chiapas & 38.32 & 891,503 & 341,624 \\
\hline
\end{tabular}

\begin{tabular}{|c|c|c|c|}
\hline Chihuahua & 41.15 & $1,600,000$ & 658,400 \\
\hline Coahuila & 49.87 & 903,194 & 450,423 \\
\hline Colima & 35.74 & 341,633 & 122,100 \\
\hline Cd. de México & 23.3 & $5,800,000$ & $1,351,400$ \\
\hline Durango & 45.33 & 636,525 & 288,537 \\
\hline Guanajuato & 37.41 & $2,000,000$ & 748,200 \\
\hline Guerrero & 47.47 & $1,200,000$ & 569,640 \\
\hline Hidalgo & 42.72 & 691,046 & 295,215 \\
\hline Jalisco & 28.82 & $3,700,000$ & $1,066,340$ \\
\hline México & 37.76 & $7,300,000$ & $2,756,480$ \\
\hline Michoacán & 52.16 & $2,600,000$ & $1,356,160$ \\
\hline Morelos & 29.09 & 790,470 & 229,948 \\
\hline Nayarit & 31.28 & 449,029 & 140,456 \\
\hline Nuevo León & 36.9 & $2,300,000$ & 848,700 \\
\hline Oaxaca & 32.53 & 665,332 & 216,432 \\
\hline Puebla & 32.85 & $1,600,000$ & 525,600 \\
\hline Querétaro & 15.18 & 725,124 & 110,074 \\
\hline Quintana Roo & 37.94 & 815,612 & 309,443 \\
\hline San Luis Potosí & 38.49 & $1,200,000$ & 461,880 \\
\hline Sinaloa & 43.66 & $1,200,000$ & 523,920 \\
\hline Sonora & 34.41 & $1,300,000$ & 447,330 \\
\hline Tabasco & 40.1 & 620,335 & 248,754 \\
\hline Tamaulipas & 55.4 & $1,200,000$ & 664,800 \\
\hline Tlaxcala & 54.12 & 663,255 & 358,954 \\
\hline Veracruz & 47.57 & $2,100,000$ & 998,970 \\
\hline Yucatán & 20.86 & 822,902 & 171,657 \\
\hline Zacatecas & 44.14 & 467,879 & 206,522 \\
\hline
\end{tabular}

Fuente: elaboración propia a partir de datos de Apple, informes de la Secretaría de Salud, e INEGI.

Nuestro interés es determinar si el cambio en el número de confirmados entre el 12 de abril y el 12 de mayo tiene una relación estadística con el número inicial de confirmados del 12 de abril y con la movilidad ponderada de esa misma fecha; es de esperar una relación positiva entre estas variables (Tabla 2).

Cuando corrimos una regresión del cambio en el número de personas confirmadas con Covid-19 entre el 12 de mayo y el 12 de abril (CAMBIOCONFIRMADOS), contra una constante, el número de casos confirmados inicial del 12 de abril (CONFIRMADOS12ABR) y un índice de movilidad del 12 de abril ponderado por el parque vehicular de cada estado del 2018 (MOVI12ABRILPONDE), se encontró un elevado ajuste en la regresión, se realizó la corrección de heteroscedasticidad de White dada la 
ISSN: 1794-9920 Impreso / Electrónico 2500-9338

Agosto- Diciembre. Volumen 20 Número 2, Año 2020 Págs. 95-106

presencia de tal problema (Tabla 2).

Tabla 2. Regresión del cambio en los confirmados de Covid-19 estatales (CAMBIOCONFIRMADOS) contra una constante $(C)$, la movilidad del 12 de abril reportada por Apple Maps ponderada por el parque vehicular estatal del 2018 (MOVI12ABRILPONDE), y el número de confirmados con Covid-19 el 12 de abril (CONFIRMADOS12ABR)

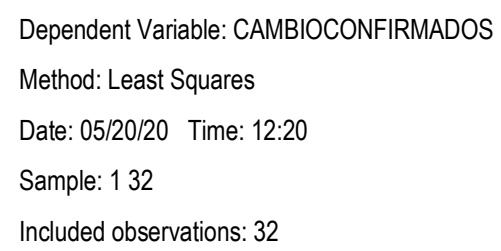

\begin{tabular}{lllll}
\hline & & & & \\
Variable & $\begin{array}{l}\text { Coefficie } \\
\text { nt }\end{array}$ & Std. Error & t-Statistic & Prob. \\
\hline & & & & \\
C & 295.7125 & 114.6455 & -2.579364 & 0.0152 \\
MOVI12ABRILPONDE & 0.000789 & 0.000179 & 4.399372 & 0.0001 \\
CONFIRMADOS12ABR & 6.305281 & 0.392995 & 16.04417 & 0.0000 \\
\hline & & & & \\
& & & 1052.2 \\
R-squared & 0.944349 & Mean dependent var & 50 \\
Adjusted R-squared & 0.940511 & S.D. dependent var & 1832.5 \\
S.E. of regression & 446.9593 & Akaike info criterion & 15.131 \\
Sum squared resid & 5793406. & Schwarz criterion & 15.269 \\
Log likelihood & 239.1100 & Hannan-Quinn criter. & 15.177 \\
F-statistic & 246.0507 & Durbin-Watson stat & 2.6658 \\
Prob(F-statistic) & 0.000000 & & 66 \\
\hline
\end{tabular}

Fuente: elaboración propia a partir de regresión, datos de Apple, informes de la Secretaría de Salud e INEGI.

Además, los residuos se distribuyen como una normal, cabe aclarar que este modelo no es dinámico y sólo está definido para un momento en el tiempo (Figura 10).
Figura 10. Prueba de normalidad de los residuales de Jarque-Bera

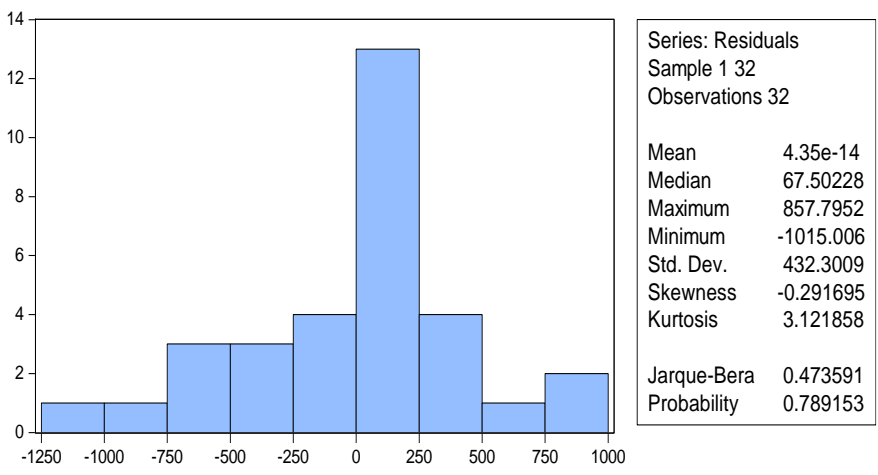

Fuente: elaboración propia a partir de regresión, datos de Apple, informes de la Secretaría de Salud e INEGI.

Ocho de 32 residuales se salieron del intervalo de confianza de +/- un error estándar (446.95): Estado de México, Morelos y Tabasco subestimados (errores positivos) y Coahuila, Jalisco, Michoacán, Nuevo León y Puebla sobreestimadas (errores negativos) (Figura 11).

Figura 11. Cambios confirmados observados (actual), cambios confirmados estimados (fitted) y diferencias entre ellos (residuals)

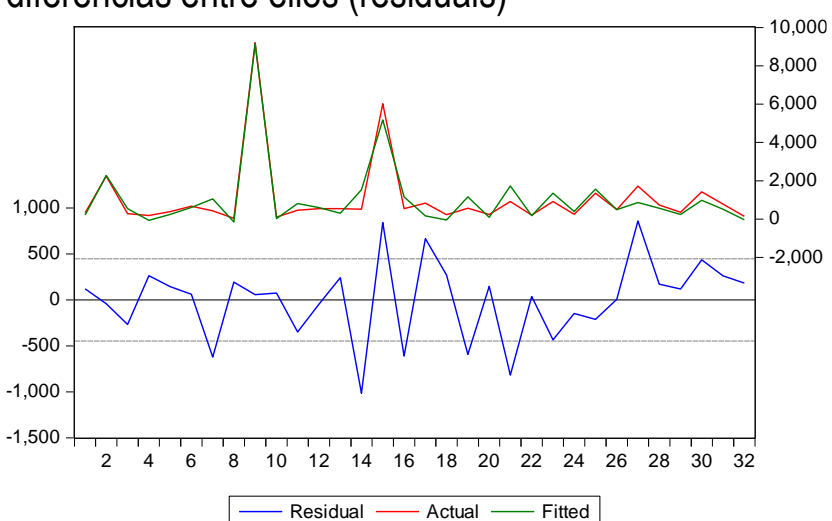

Fuente: elaboración propia a partir de regresión, datos de Apple, informes de la Secretaría de Salud e INEGI.

\section{¿CÓMO FINANCIAR UNA POLÍTICA DE CONFINAMIENTO Y UN POSTERIOR APOYO A LA DEMANDA AGREGADA?}

El problema para la reactivación es una cuestión de sincronización: cada lugar en México va a su ritmo y en diferentes fases de la epidemia, para lograr reducir la transmisión se debe reducir la movilidad, por ello es necesario brindar condiciones para que diferentes grupos de 
empresas y trabajadores disminuyan su actividad, y así los contagios; para en su momento iniciar e implementar una política de reactivación económica.

Una política de esta naturaleza debe identificar y definir la situación actual, cómo será financiada, a quién beneficiará, con qué montos.

\section{¿Cómo financiar estos programas de apoyo para el confinamiento y, en su momento, la reactivación?}

Con el empleo de la una parte de la Línea de Crédito Flexible del Fondo Monetario Internacional, que fue renovada en el último trimestre de 2019 por el Secretario de Hacienda y crédito Público (SHCP) por un monto de 60 mil millones de dólares; un crédito a pagar con recursos del remanente de Banco de México a entregar a finales de marzo de 2021, gran parte del cual se debe a las ganancias cambiarias sobre las reservas (capital contable).

En 2015 Agustín Carstens, entonces Gobernador del Banco de México, entregó al gobierno federal un remanente de operación por 239.064 mil millones de pesos, esencialmente conformado por las ganancias cambiarias de ese año, en el cual el tipo de cambio inició en 14.98 pesos por dólar y terminó en 17.18 pesos por dólar, con una depreciación de 2.2 pesos, 14.68 por ciento. Las reservas internacionales estaban al 31 de diciembre de 2014 en 193 mil 239 millones de dólares, para el 31 de diciembre de 2015 se registró una reserva por 176 mil 723 millones de dólares; en otras palabras, tales remanentes significaron $1.28 \%$ del PIB de 2015. Cabe agregar que en 2017 Banco de México entregó remanentes por 321.653 mil millones de pesos, que representaron 1.55 por ciento del PIB de ese año; estos remanentes dependen del capital contable y otros pasivos de esa institución.

Tal remanente puede ascender, según nuestras estimaciones, aproximadamente a 547 mil 735 millones de pesos, si consideramos unas reservas promedio anual de 180 mil millones de dólares y una depreciación de $25 \%$ a lo largo de 2020 . Un cálculo, bajo supuestos débiles, para el remanente donde consideramos una depreciación mínima del $25 \%$ y un monto similar de reservas internacionales (promedio anual de 185 mil millones de dólares); y el capital contable, que al 8 de mayo de este año registra 894,900 millones de pesos, nos indica un monto de remanente, con ganancias cambiarias, de 547 mil 735 millones de pesos, que representaría 2.08 por ciento del PIB estimado por el Gobierno Federal para el 2020 (Tabla 3).

Uno de los problemas que se enfrentará con los programas de reactivación económica es que se agotarán a sí mismos por no ser un esfuerzo sincronizado a nivel nacional. La probabilidad de éxito de reducir los contagios y muertes, gracias a disminuir la movilidad al otorgar recursos para apoyar económicamente a quienes se aíslen, cuyos recursos se pueden pagar en un corto plazo, es una oportunidad para aprovechar. Otra parte de los recursos del crédito se podría emplear para la reactivación económica.

Con los recursos del remanente a entregar en 2021 se debe cumplir lo establecido en la Ley Federal de Presupuesto y Responsabilidades Hacendarias.

Tabla 3. Variables a considerar para calcular el remanente que entrega Banxico al Gobierno Federal, 2015-2020

\begin{tabular}{|c|c|c|c|c|c|c|}
\hline Fecha & $\begin{array}{l}\text { Tipo de } \\
\text { cambio } \\
\text { a inicio } \\
\text { de año }\end{array}$ & $\begin{array}{l}\text { Tipo de } \\
\text { cambio } \\
\text { a final } \\
\text { de año }\end{array}$ & $\begin{array}{l}\text { Variación } \\
\text { (pesos) }\end{array}$ & $\begin{array}{l}\text { Variación } \\
(\%)\end{array}$ & $\begin{array}{l}\text { Otros pasivos y } \\
\text { capital contable, } \\
\text { neto de otros } \\
\text { activos (millones } \\
\text { de pesos) }\end{array}$ & $\begin{array}{l}\text { Reservas } \\
\text { internaciona } \\
\text { les (millones } \\
\text { de dólares) }\end{array}$ \\
\hline 2015 & $\begin{array}{l}14.82 \\
9\end{array}$ & $\begin{array}{l}17.24 \\
87 \\
\end{array}$ & 2.4197 & $16.32 \%$ & 535,473 & 176,723 \\
\hline 2016 & $\begin{array}{l}17.35 \\
29\end{array}$ & $\begin{array}{l}20.61 \\
94\end{array}$ & 3.2665 & $18.82 \%$ & 834,821 & 176,542 \\
\hline 2017 & $\begin{array}{l}20.73 \\
23 \\
\end{array}$ & 19.66 & -1.0723 & $-5.17 \%$ & 259,630 & 172,802 \\
\hline 2018 & $\begin{array}{l}19.48 \\
99\end{array}$ & 19.65 & 0.1601 & $0.82 \%$ & 167,526 & 174,793 \\
\hline 2019 & $\begin{array}{l}19.58 \\
78\end{array}$ & $\begin{array}{l}18.84 \\
52\end{array}$ & -0.7426 & $-3.79 \%$ & -990 & 181,177 \\
\hline $\begin{array}{l}2020 \\
*\end{array}$ & $\begin{array}{l}18.88 \\
17\end{array}$ & 23.61 & 4.7283 & $25.04 \%$ & $894,900^{*}$ & $186,643^{*}$ \\
\hline
\end{tabular}

\footnotetext{
*: Datos al 8 de mayo

**: Datos supuestos al final de año

Fuente: Banxico. Tipos de cambio/Mercado cambiario.

Banxico. Estado de cuenta semanal.

Estados de cuenta del Banco de México de la última semana de los años 2015-2019 y al 8 de mayo de 2020.
} 


\section{CONCLUSIONES:}

Existe una relación estadística entre el incremento en los casos de contagios de COVID-19 y la movilidad; por ende, la finalización de la llamada por el gobierno federal Jornada de sana Distancia tendráXie, Z., Qin, Y., Li, Y., Li, Y., Shen, W., Zheng, Z., \& Liu, S. implicaciones en los contagios y en las muertes por COVID-19. Una política de apoyo a los trabajadores formales e informales y a las micro, pequeñas y medianas empresas, así como para la reactivación económica puede ser financiado con recursos de un crédito del Fondo Monetario Internacional a ser cubierto con los remanentes que Banco de México entregará al gobierno federal en 2021.

La Ley de Banco de México señala en el Artículo 55 el Banco deberá entregar al Gobierno Federal el importe íntegro de su remanente de operación una vez constituidas las reservas previstas en esta Ley, siempre que ello no implique la reducción de reservas provenientes de la revaluación de activos, con fecha límite en abril del ejercicio inmediato siguiente. En este sentido lo que podría hacer el Gobierno federal es buscar acelerar la entrega de dichos recursos a principios del 2021.

Por su parte, en la Ley Federal de Presupuesto y Responsabilidades Hacendarias en su artículo 19 bis señala que cuando menos el setenta por ciento de este remanente se deberá destinar a la amortización de la deuda pública del Gobierno Federal contratada en ejercicios fiscales anteriores o a la reducción del monto de financiamiento necesario para cubrir el Déficit Presupuestario que, en su caso, haya sido aprobado para el ejercicio fiscal en que se entere el remanente, o bien, una combinación de ambos conceptos. Pero si se hace lo anterior ello no tendrá efectos sobre la demanda agregada, de ahí otro de los motivos para la propuesta planteada en este estudio.

El resto de estos recursos deberá ir, por ley, a fortalecer el Fondo de Estabilización de los Ingresos Presupuestarios 0 al incremento de activos que fortalezcan la posición financiera del Gobierno Federal.

\section{REFERENCIAS:}

Apple Maps. Informes de tendencia de movilidad Carteni, A., Di Franceso, L., \& Martino, M. (2020). How mobility habits influenced the spread of the COVID-19 pandemic: Results from the Italian case study. Science of the Total Environment(741), 1-9.
Google Community Mobility Reports. Informes de movilidad local sobre el Covid-19 Secretaría de Salud. Informes de contagiados por entidad federativa. (2020). Spatial and temporal differentiation of COVID19 epidemic spread in mainland China and its influencing factors. Science of the Totl Enviroment(744), 1-9. 